\title{
BMJ Open Chewing gum to treat postoperative nausea and emesis in female patients (CHEWY): rationale and design for a multicentre randomised trial
}

\author{
Jai Darvall, ${ }^{\oplus 1,2}$ Britta Sylvia von Ungern-Sternberg, ${ }^{3,4}$ Sabine Braat, ${ }^{5}$ David Story, ${ }^{2}$ \\ Andrew Davidson, ${ }^{6,7}$ Megan Allen, ${ }^{1,2}$ An Tran-Duy, ${ }^{8}$ Dana Middleton, ${ }^{1}$ Kate Leslie ${ }^{1,2}$
}

To cite: Darvall J, von Ungern-Sternberg BS, Braat $\mathrm{S}$, et al. Chewing gum to treat postoperative nausea and emesis in female patients (CHEWY): rationale and design for a multicentre randomised trial. BMJ Open 2019;9:e027505. doi:10.1136/ bmjopen-2018-027505

- Prepublication history for this paper is available online. To view these files please visit the journal online (http://dx.doi org/10.1136/bmjopen-2018027505).

Received 25 October 2018 Revised 21 March 2019 Accepted 2 May 2019
Check for updates

(C) Author(s) (or their employer(s)) 2019. Re-use permitted under CC BY-NC. No commercial re-use. See rights and permissions. Published by BMJ.

For numbered affiliations see end of article.

Correspondence to

Dr Jai Darvall;

jai.darvall@mh.org.au

\section{ABSTRACT}

Introduction Postoperative nausea, retching and vomiting (PONV) remains one of the most common side effects of general anaesthesia, contributing significantly to patient dissatisfaction, cost and complications. Chewing gum has potential as a novel, drug-free alternative treatment. We aim to conduct a large, definitive randomised controlled trial of the efficacy and safety of peppermint-flavoured chewing gum to treat PONV in the postanaesthesia care unit (PACU). If chewing gum is shown to be as effective as ondansetron, this trial has the potential to significantly improve outcomes for tens of millions of surgical patients around the world each year.

Methods and analysis This is a prospective, multicentre, randomised controlled non-inferiority trial. 272 female patients aged $\geq 12$ years having volatile anaesthetic-based general anaesthesia for breast or laparoscopic surgery will be randomised. Patients experiencing nausea, retching or vomiting in PACU will be randomised to $15 \mathrm{~min}$ of chewing gum or $4 \mathrm{mg}$ intravenous ondansetron. The primary outcome (complete response) is cessation of PONV within 2 hours of administration, with no recurrence nor rescue medication requirement for 2 hours after administration. Ethics and dissemination The Chewy Trial has been approved by the Human Research Ethics Committees at all sites. Dissemination will be via international and national anaesthesia conferences, and publication in the peerreviewed literature.

Trial registration number ACTRN12618000429257; Preresults.

\section{INTRODUCTION}

More than 300 million patients have surgery each year worldwide, with the greatest growth in recent years in low-income nations. ${ }^{1}$ Postoperative nausea, retching and vomiting (PONV) affects up to $80 \%$ of untreated patients after general anaesthesia, females disproportionately more than males, and contributes significantly to patient discomfort, costs of care and potentially life-threatening complications such as aspiration and wound dehiscence. $^{2-6}$ PONV is independently associated with a tripled incidence of admission

\section{Strengths and limitations of this study}

- This is the first large randomised trial designed to evaluate the impact of chewing gum on postoperative nausea and vomiting.

- The trial is designed as a multicentre, international study, enhancing external validity.

- The inclusion of health economic and treatment acceptability assessments is a strength of this trial.

- Limitations include the inability to blind patients and postanaesthesia care unit nurses to group allocation, and restriction to patients aged $\geq 12$ years (potentially reducing applicability to younger children).

after scheduled day surgery compared with no PONV, and has been the leading cause of unplanned admission in patients undergoing ambulatory gynaecological surgery. ${ }^{78}$ In children, PONV accounts for $25 \%$ of admissions following scheduled day surgery. ${ }^{9}$ PONV leads to increased costs of treatment and hospital care, increased length of stay in the postanaesthesia care unit (PACU) and logistical difficulties for hospitals, patients and their families. ${ }^{10}$ PONV remains one of the most common reasons for patient dissatisfaction with anaesthesia, ${ }^{11}$ and patients are willing to pay up to US $\$ 100$ to avoid PONV. ${ }^{12}$

Numerous guidelines on the prophylaxis and treatment of PONV have been published. The Consensus Guidelines published by the Society for Ambulatory Anesthesiology provide the most authoritative summary of the evidence. ${ }^{3}$ Despite their wide and longstanding promulgation, and a wealth of PONV literature, evidence suggests poor compliance with the Guidelines, especially in low-income nations. ${ }^{61013}$ In one study of over 23000 anaesthetics, only $30 \%$ of high risk patients received the recommended antiemetic prophylaxis. ${ }^{14}$ Therefore, PONV remains a significant problem that requires 
treatment. When PONV occurs, with or without prophylaxis, the Guidelines recommend treatment with a range of first-line drugs such as $5-\mathrm{HT}_{3}$ receptor antagonists (eg, ondansetron) and butyrophenones (eg, droperidol). These drugs have side effects, for example prolonged QT interval, headache, elevated liver enzymes and constipation. ${ }^{3}$ Additionally, the combination of large numbers of patients treated and treatment failures can be costly, particularly in low-income nations in which surgical volumes are rapidly rising $(115 \%$ increase in surgery rate between 2004 and 2012 for countries with per capita health expenditure $<$ US $\$ 400$ per annum ${ }^{1}$ ). The cost of antiemetic drugs may be paradoxically higher in these regions, for example, while ondansetron costs US $\$ 0.15$ in Australia, it costs US\$1.11 in the Sudan, about $2 \%$ of the total annual per capita health spend in that nation. ${ }^{15}$

To minimise both harm and cost, there is emerging interest in drug-free alternative treatments for PONV. Acupuncture at the $\mathrm{P} 6$ point on the inner forearm ${ }^{16}$ and ginger ${ }^{17}$ have proved efficacious in preventing PONV compared with placebo, and acustimulation has been reported as equivalent to ondansetron for treatment. ${ }^{18}$ These modalities have not seen widespread uptake, however, due to clinician unfamiliarity, and training and equipment requirements. A simple drug- and equipment-free treatment—chewing gum-has been found to be efficacious for the related problem of postoperative paralytic ileus following gastrointestinal surgery. Hypothesised mechanisms of its effect include 'sham feeding', with increased gastrointestinal activity mediated via cephalic-vagal stimulation from chewing. A 2015 meta-analysis including 9072 patients across 81 randomised controlled trials demonstrated evidence for reduced postoperative ileus and earlier hospital discharge ${ }^{19}$ We hypothesise that mechanisms underpinning the efficacy of chewing gum in the resolution of postoperative ileus may have a role in the treatment of PONV.

\section{Feasibility}

We assessed the feasibility of chewing gum in an initial prospective cohort study, which enrolled 41 female patients having gynaecological laparoscopy, with a median age of 31 (range $18-52$ ) years. ${ }^{20}$ Thirty-one patients $(76 \%)$ reached an Observer's Assessment of Alertness and Sedation (OAA/S) score of $5,{ }^{21}$ and successfully completed a period of gum chewing in the PACU (median duration $15 \mathrm{~min}$, range $3-40 \mathrm{~min}$ ). There were no identified safety concerns with only one piece of gum intentionally swallowed and none inhaled, and chewing gum was found to be acceptable to patients and PACU nurses alike. Our subsequent randomised controlled pilot study ${ }^{22}$ enrolled 94 female patients undergoing breast or laparoscopic surgery who received intraoperative antiemetic prophylaxis, and randomised them to intravenous ondansetron $4 \mathrm{mg}$ or chewing gum if nausea, retching and/or vomiting was experienced in the PACU. Feasibility was demonstrated, as recruitment was satisfactory, the protocol was acceptable to anaesthetists and nurses, and data collection was complete. Twenty-eight of the 94 patients (30\%) developed nausea, retching and/ or vomiting in the PACU (13 randomised to ondansetron; 15 randomised to chewing gum, of whom two were insufficiently awake and one refused to chew gum). Using a hypothesised difference for equivalence of $15 \%$, on a per-protocol basis (preferred methodology for testing a non-inferiority hypothesis ${ }^{23}$ ), complete response of the first episode of nausea, retching and/or vomiting without requirement for rescue medication occurred in 9 of 12 $(75 \%)$ of chewing gum patients versus 5 of $13(38.5 \%)$ of ondansetron (risk difference $37 \%, 90 \%$ CI $6 \%$ to $67 \%$, $\mathrm{p}=0.07$ ). The intention-to-treat findings were consistent with the per-protocol results.

We aim to demonstrate the non-inferiority of this simple and inexpensive treatment compared with ondansetron for the common and distressing problem of PONV in a multicentre, randomised controlled trial. This study has the potential to introduce a novel drug-free treatment for a common complication of anaesthesia and surgery, without the side effects and cost associated with conventional drug treatment. As such, it has potential to significantly improve health outcomes and save money for millions of patients and health services in high-income, middle-income and low-income nations worldwide.

\section{Study hypotheses}

Our primary hypothesis is that chewing gum is non-inferior to ondansetron in achieving complete cessation of nausea, retching and vomiting within 2 hours of administration, with no recurrence between cessation and 2 hours after administration, and no rescue medication between administration and 2 hours after administration (ie, complete response), in female patients aged $\geq 12$ years with nausea, retching and/or vomiting in the PACU after volatile anaesthetic-based general anaesthesia for breast or laparoscopic surgery. Our secondary hypotheses are that chewing gum is superior to ondansetron with respect to: (1) complete response (as defined above), if chewing gum is found to be non-inferior; (2) acceptability of randomised treatment to patients and PACU nurses; (3) time to complete response in the 2 hours after randomised treatment; (4) numbers of episodes of nausea, retching and/or vomiting in the 2 hours after randomised treatment; (5) numbers of rescue treatments for nausea, retching and/or vomiting in the 2 hours after randomised treatment; (6) duration of PACU stay; (7) quality of recovery; (8) functional health and well-being; (9) duration of hospital stay from PACU admission; (10) costs of randomised medications, rescue medications and hospital stay and (11) willingness-to-pay to achieve the primary outcome.

\section{METHODS AND ANALYSIS}

\section{Study design and setting}

The Chewy Trial is a multicentre, randomised, international, parallel-group, non-inferiority trial, with patients 
randomised equally to either chewing gum or ondansetron. Patients will be recruited from approximately 20 adult and paediatric hospitals across Australia, New Zealand and Hong Kong affiliated with the Australian and New Zealand College of Anaesthetists Clinical Trials Network. A block permuted randomisation list stratified by site will be computer-generated by an independent statistician. Allocation to treatment arms will be carried out centrally by a computer. Recruitment commenced in July 2018, and is expected to be completed in early 2020 .

\section{Inclusion criteria}

The following patients are eligible for enrolment:

- Written informed consent (patient consent, parent/ guardian consent).

- Female sex.

- Aged 12 years and older.

- Weight $\geq 30 \mathrm{~kg}$.

- Volatile anaesthetic-based general anaesthesia.

- Preoperative Apfel score $\geq 2$.

- Breast or laparoscopic surgery.

\section{Exclusion criteria}

The following exclusion criteria apply:

- Plan to use propofol-maintained general anaesthesia.

- Plan to use inhalational induction of general anaesthesia without propofol coinduction.

- Contraindication to chewing gum:

- Impaired pharyngeal/oesophageal function (eg, bulbar palsy, achalasia).

- Phenylketonuria (contraindication to sweetener aspartame in chewing gum).

- Full upper and/or lower denture (not feasible to chew gum).

- Contraindication to any protocolised antiemetic drug (prophylaxis, randomised intervention or rescue).

- Treatment with any of the study antiemetics within 8 hours of induction.

- Planned postoperative mechanical ventilation.

\section{Study procedures}

\section{Intraoperative management}

Patient monitoring will be established in accordance with the standards of the Australian and New Zealand College of Anaesthetists. ${ }^{24}$ If indicated midazolam and/ or clonidine may be administered as premedication or during induction. General anaesthesia will be induced with propofol and an opioid; coinduction with sevoflurane is permitted. If indicated neuromuscular blockade will be established. After placement of either a supraglottic airway or endotracheal tube, anaesthesia will be maintained with sevoflurane in oxygen and/or air. Nitrous oxide will be prohibited during the maintenance phase. Further opioid and/or non-opioid analgesia and/ or regional or local anaesthesia will be administered at the discretion of the attending anaesthetist. Intravenous fluids, prophylactic antibiotics and venous thromboembolism prophylaxis will be administered according to local protocols. Patient core temperature $>36^{\circ} \mathrm{C}$ will be targeted using assisted warming devices.

Antiemetic prophylaxis will be administered intraoperatively according to PONV risk, as determined by the investigators using the Apfel risk score and commensurate with the Consensus Guideline. ${ }^{3}$ Patients with 2-3 risk factors ('medium risk') will receive one drug, dexamethasone $4 \mathrm{mg}$ intravenous [recommended dose of dexamethasone is $0.15 \mathrm{mg} / \mathrm{kg}$ ( $4 \mathrm{mg}$ in a $27 \mathrm{~kg}$ patient with all recruited patients $\geq 30 \mathrm{~kg}$ ) ]; and patients with 4 risk factors ('high risk') will receive two drugs, dexamethasone $4 \mathrm{mg}$ intravenous and droperidol $10 \mathrm{mcg} / \mathrm{kg}$ to a maximum of $0.625 \mathrm{mg}$ intravenous. This protocol is familiar to and easily employed by anaesthetists. At the end of surgery, and if applicable, neuromuscular blockade will be reversed with neostigmine and glycopyrrolate or atropine, or sugammadex. When appropriate, patients will be transferred to the PACU. Anaesthetists will record intraoperative data on the case report form (see below).

\section{Postoperative management}

Postoperative pain will be managed at the discretion of the attending anaesthetist. Antiemetics other than the randomised intervention and rescue medications will be prohibited. Patients will be observed from their arrival in the PACU by a blinded observer who is a member of the research team. While in the PACU, patients may spontaneously report nausea or be observed to retch or vomit. If patients do not spontaneously report nausea and are not observed to retch or vomit, they will be asked 'Are you feeling sick?' every $15 \mathrm{~min}$ by the observer. If nausea is present, its severity will be recorded as mild, moderate or severe. ${ }^{25}{ }^{26}$ If nausea, retching and/or vomiting are present the patient will be assessed for alertness using the $\mathrm{OAA} / \mathrm{S}$ score (figure 1 ) ${ }^{21}$ If the score is 5 , the observer will leave the PACU, to maintain blinding to group allocation. The PACU nurse will randomise the patient using REDCap, ${ }^{27}$ an electronic data capture tool hosted at The University of Melbourne, and then administer the randomly allocated intervention. Allocation concealment will be maintained, as the module will not release the randomised group assignment until after patient recruitment, completion of baseline measurements, and PONV in PACU is experienced. The PACU nurse will encourage the patient to chew the chewing gum (if applicable) for the full $15 \mathrm{~min}$ period, after which it will be discarded, and the patient and PACU nurse will rate acceptability of the randomised treatment. This interval has been chosen to encompass the peak effect of ondansetron $(10 \mathrm{~min})^{28}$ and also the time to complete response seen with chewing gum in our pilot trial [median (25th-75th percentile) time to complete response $10(7-15) \mathrm{min}]{ }^{22}$ The blinded observer will then return to the PACU to observe the patient continuously for 2 hours after randomisation, noting the time of resolution of the index episode of PONV, and any recurrent retching, vomiting or nausea. This observation period will continue on the 


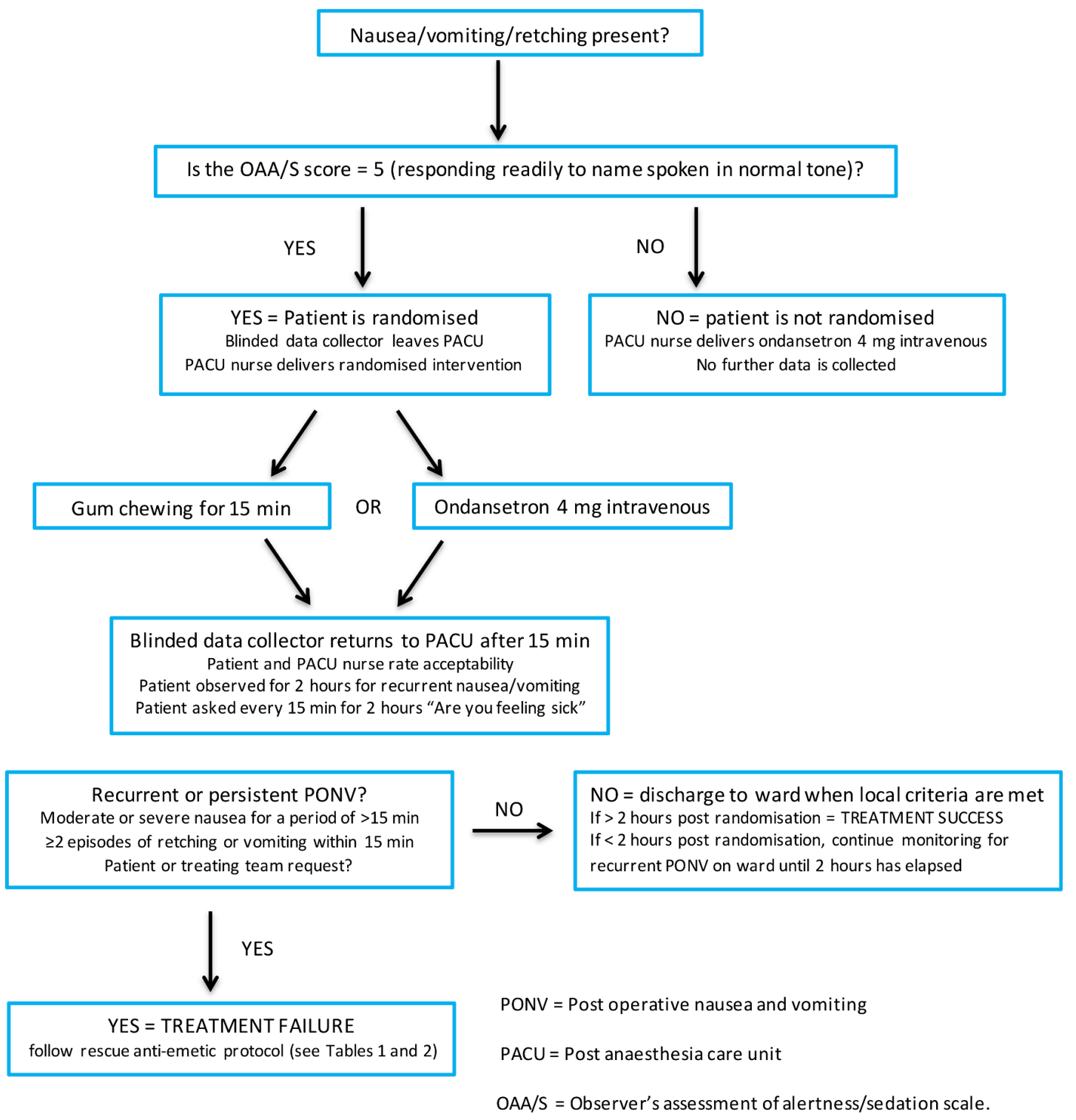

Figure 1 Management of PONV in PACU.

ward if the patient is discharged from the PACU prior to 2 hours elapsing.

If the OAA/S score is $<5$, chewing gum will not be allowed and thus the patient will not be randomised. The patient will receive antiemetic treatment at the discretion of the attending anaesthetist and will not be followed further. Patients who are randomised to chewing gum but who do not chew it (for whatever reason) will receive ondansetron $4 \mathrm{mg}$ intravenous (the first rescue treatment in the chewing gum group) and will continue in the study as part of the chewing gum intention-to-treat set.

Rescue medication will be administered to patients who report a nausea score of moderate or severe for a period of $>15 \mathrm{~min}, \geq 2$ episodes of retching or vomiting within $15 \mathrm{~min}$, at patient request or at the discretion of the treating team any time from the administration of the randomised treatment to 2 hours later (tables 1 and 2). The observer will be blind to the identity of each rescue medication given. Patients will be discharged from the PACU when they meet local discharge criteria; if discharge occurs prior to 2 hours elapsing during which no nausea, retching and/or vomiting are present, then the patient will be ineligible for randomisation.

\section{Blinding}

Blinding of patients, parents/guardians (if present in the PACU) and PACU nurses to the randomised intervention will not be possible given the nature of the intervention. Patients, parents/guardians and PACU nurses will be advised of their role in promoting observer blinding. Blinding of observers who collect data in the PACU will be facilitated by the observer departing prior to randomisation and returning after removal of chewing gum and rating of acceptability, thus being unaware of group allocation. Observers will be blind to the identity of rescue medications, so that they will not be alerted to group allocation. The difficulty of full blinding is a frequent 
Table 1 Rescue antiemetic treatment (patients aged $\geq 18$ years)

\begin{tabular}{lllll}
\hline Intraoperative & Intervention & Rescue 1 & Rescue 2 & Rescue 3 \\
\hline Dexamethasone & Chewing gum & Ondansetron 4 mg & Droperidol 0.625 mg & Cyclizine 50 mg \\
& Ondansetron 4 mg & Droperidol 0.625 mg & Cyclizine 50 mg & Metoclopramide 20 mg \\
Dexamethasone+droperidol & Chewing gum & Ondansetron 4 mg & Cyclizine 50 mg & Metoclopramide 20 mg \\
& Ondansetron 4 mg & Cyclizine 50 mg & Metoclopramide 20 mg & Propofol 20 mg \\
\hline
\end{tabular}

All medications apart from propofol can be administered on the ward if patient is discharged from PACU $<2$ hours postrandomisation, still within the observation period. All medications administered intravenously.

methodological issue in trials involving non-pharmacological antiemetic strategies, and previous studies examining modalities such as acustimulation and aromatherapy have been completely unblinded. ${ }^{18} 25$ The study statistician, data collectors, outcome assessors, data analysts and manuscript writers will remain blinded until database lock.

\section{Data and safety monitoring}

An independent Data and Safety and Monitoring Committee (DSMC) comprised of an experienced academic anaesthetist (chair), another academic anaesthetist and an independent epidemiologist/statistician will meet regularly to review blinded trial data and safety endpoints in its open sessions and have access to unblinded group and patient data in its closed sessions. Given the benign nature of the study interventions (chewing gum and ondansetron) and the common but mild nature of PONV, no interim analyses to stop the trial early are planned. The DSMC may request unplanned interim analyses.

\section{Primary outcome}

The primary outcome of the trial is complete cessation of nausea, retching and vomiting within 2 hours of administration, with no recurrence between cessation and 2 hours after administration, and no rescue medication between administration and 2 hours after administration (ie, complete response).

\section{Secondary outcomes}

Planned secondary outcomes are:

1. Acceptability of randomised treatment to patients and PACU nurses.

2. Time to complete response in the 2 hours after randomised treatment.

3. Numbers of episodes of nausea, retching and/or vomiting in the 2 hours after randomised treatment.

4. Numbers of rescue treatments for nausea, retching and/or vomiting in the 2 hours after randomised treatment ${ }^{29}$.

5. Duration of PACU stay.

6. Quality of recovery (using the QoR-15 score),${ }^{29} 24$ hours after randomisation or at hospital discharge, whichever is sooner.

7. Functional health and well-being (using the SF-12 or SF-10 paediatric), ${ }^{30} 24$ hours after randomisation or at hospital discharge, whichever is sooner.

8. Duration of hospital stay from PACU admission.

9. Costs of randomised medications, rescue medications and hospital stay.

10. Willingness-to-pay to achieve the primary outcome.

Table 2 Rescue antiemetic treatment (patients aged $<18$ years)

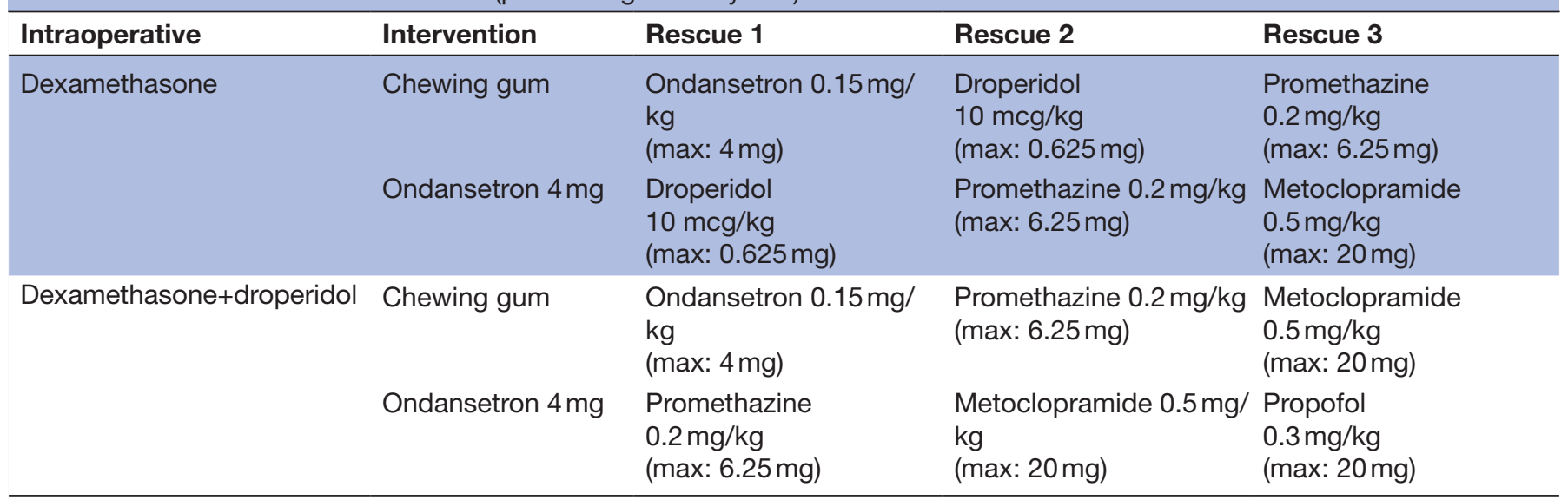

All medications apart from propofol can be administered on the ward if patient is discharged from PACU $<2$ hours postrandomisation, still within the observation period. All medications administered intravenously. 


\section{Safety outcomes}

The following safety outcomes occurring between randomisation and 24 hours after randomisation or hospital discharge (whichever is sooner) will be reported:

1. Swallowing of chewing gum.

2. Inhalation of chewing gum.

3. Unplanned overnight admission for scheduled day cases.

4. Unplanned intensive care unit admission.

5. Cardiovascular events (myocardial infarction, stroke).

6. Pulmonary events (postoperative pulmonary complications).

7. Wound events (bleeding, dehiscence).

8. Death.

\section{Sample size}

In our pilot study, ${ }^{22}$ complete response of the first episode of nausea, retching and/or vomiting without requirement for rescue medication occurred in 9 of 12 $(75 \%)$ of chewing gum patients versus 5 of $13(38 \%)$ of ondansetron (risk difference $37 \%, 90 \%$ CI $6 \%$ to $67 \%$, $\mathrm{p}=0.07)$. Excluding two of these chewing gum patients and one ondansetron patient who had a recurrent episode of PONV in the PACU after complete response, 7 of the 12 (58\%, Wilson's $95 \%$ CI $32 \%$ to $81 \%$ ) chewing gum patients and 4 of the 13 (31\%, Wilson's 95\% CI $13 \%$ to $58 \%$ ) ondansetron patients achieved complete response without recurrence. The risk difference was $28 \%$ (Newcombe's $95 \%$ CI $-5 \%$ to $53 \%$ ). Assuming $40 \%$ of patients will achieve complete response without recurrence in the ondansetron group (conservative) and 50\% in the chewing gum group (numerically $10 \%$ better than ondansetron), a sample size of 129 patients per arm (258 in total) will be required to provide valid data for the per-protocol analysis to establish non-inferiority by having the lower limit of the two-sided 95\% CI of the difference between chewing gum and ondansetron higher than $-10 \%$ with $90 \%$ power. Anticipating $5 \%$ of patients violating the per-protocol definition and thus excluded from the primary analysis set, the total number of patients to be randomised is 272 .

The non-inferiority margin was obtained using the statistical fixed-margin (95\%-95\% method) approach ${ }^{31}$ combined with expert opinion. For the fixed margin approach, a random effects meta-analysis of three doubleblind randomised studies evaluating the effect of ondansetron versus placebo on complete response of PONV for 2 hours after administration was used. ${ }^{32-34}$ Data across different doses of ondansetron were pooled, which is clinically acceptable given that a previous systematic review concluded lack of evidence of a clinically relevant dose-response pattern. ${ }^{35}$ This meta-analysis resulted in an estimate of $37 \%(95 \%$ CI $26 \%$ to $48 \%)$ in favour of ondansetron over placebo which led to a clinical margin of $13 \%$ (ie, $50 \%$ of the lower limit of the $95 \%$ CI). The clinical expert opinion approach started with the assumption that the proportion of patients achieving complete response without any treatment will be substantially lower than that observed with ondansetron. Across the three historical studies ${ }^{32-34}$ this proportion ranged from $8 \%$ to $34 \%$ in the placebo arms compared with $49 \%$ to $78 \%$ in the ondansetron arms and ondansetron showed consistent superior efficacy to placebo. Using our definition of the primary outcome, we anticipate that the difference between ondansetron and placebo would have been at least $20 \%$ in favour of ondansetron if our study included a placebo arm. Therefore, the use of a non-inferiority margin of at most $10 \%$ is considered a valid approach for evaluating chewing gum compared with ondansetron. Combining these two approaches, the non-inferiority margin was set to $10 \%$.

\section{Statistical analyses}

The primary analysis of the primary outcome of complete response will be based on the per-protocol set (ie, all randomised patients receiving the treatment they were randomised to and who provide valid primary outcome data). A sensitivity analysis will be conducted for the intention-to-treat set (ie, all randomised patients according to their randomised treatment).

A generalised linear model with identity link and binomial distribution will be fitted to the primary outcome, with treatment group and site as independent variables. Non-inferiority of chewing gum to ondansetron will be concluded if the lower limit of the two-sided $95 \%$ CI for the absolute difference in the proportion of patients achieving complete response is greater than $-10 \%$ for the per-protocol set. If non-inferiority is demonstrated, superiority of chewing gum over ondansetron will be concluded if the same limit is greater than $0 \%$ for the intention-totreat set. In addition, we will obtain an adjusted treatment effect accounting for type of surgery (breast and laparoscopic). Sensitivity analyses will be performed to explore the potential impact of missing data on the results of the primary outcome for the intention-to-treat set. Analysis of the secondary outcomes will follow the intention-totreat principle and superiority testing will be conducted of chewing gum versus ondansetron. A Cox proportional hazards model will be fitted to the time to complete response, with the underlying proportional hazard assumption tested using the scaled Schoenfeld residuals. Quality of recovery and functional health and well-being continuous outcomes will be analysed using linear regression models with baseline value, treatment group and site in the model. Count data (number of episodes of nausea, retching and vomiting, number of rescue antiemetics) and length of stay (duration of PACU stay and duration of hospital stay) will be modelled using generalised linear regression models. Safety outcomes will be summarised according to treatment received. Subgroup analyses will include assessment by age ( $<18 \mathrm{vs} \geq 18$ years), type of surgery (breast vs laparoscopic), and by Aboriginal and Torres Strait Islander and Māori status (yes vs no) and will be evaluated by including the interaction between treatment group and subgroup into the model. 


\section{Health economic analyses}

Health economic analyses will be based on the comparison between the chewing gum and ondansetron groups in terms of total costs and proportions of patients with the primary outcome. Cost incurred by each patient in each treatment arm will be calculated based on resource utilisation (quantities of chewing gum or ondansetron and medications used in rescue treatment, and length of hospital stay), and unit costs. Unit price for chewing gum will be based on the market price in Victoria, and unit costs for medications on the price list of the Pharmaceutical Benefit Scheme. Costs of hospital admissions, adjusted for the length of hospital stay, will be calculated using the diagnosis-related groups, cost weights ((Weighted Inlier Equivalent Separation (WIES)) and WIES prices published by Victoria State Government. In line with the primary and secondary hypotheses, we expect that chewing gum will be dominant over ondansetron, ie, the average cost per patient will be lower and the probability that a patient achieves the primary outcome in the chewing gum group will be higher compared with the ondansetron group. If the chewing gum treatment is found not to be dominant, the incremental cost of treatment with chewing gum to have one more patient achieving the primary outcome compared with treatment with ondansetron (ie, incremental cost-effectiveness ratio (ICER)), will be calculated. If the baseline risk factors are not similar between the two treatment groups, costs and health outcomes will be adjusted for these factors using the statistical model described in the previous section for health outcome and log-normal regression models for costs. If there are missing data, multiple imputation combined with bootstrap inference will be used to capture uncertainty surrounding the ICER. Mean willingness-to-pay will be calculated and compared with the ICER to inform the decision makers whether treatment with chewing gum is cost effective. We will perform internal validation of the willingness-to-pay by checking if there will be a positive influence of health outcomes and income on these measures.

\section{Patient and public involvement}

We have involved an experienced health consumer advocate at all stages of the project, from protocol development to ethics application and trial conduct. The health consumer advocate is a member of the Trial Steering Committee.

\section{ETHICS AND DISSEMINATION}

Results from this study will be published in a peer-reviewed medical journal and presented at national and/or international anaesthesia conferences.

\section{CONCLUSION}

This trial will provide definitive evidence as to whether chewing gum is non-inferior to ondansetron for the management of postoperative nausea and vomiting after volatile-based general anaesthesia in female patients. This trial has the potential to significantly improve clinical and economic outcomes for millions of patients and health services worldwide.

\section{Author affiliations}

${ }^{1}$ Department of Anaesthesia and Pain Management, Royal Melbourne Hospital, Melbourne, Victoria, Australia

${ }^{2}$ Centre for Integrated Critical Care, University of Melbourne, Melbourne, Victoria, Australia

${ }^{3}$ Department of Anaesthesia and Pain Management, Perth Children's Hospital, Perth, Western Australia, Australia

${ }^{4}$ Anaesthesia Unit, Medical School, University of Western Australia, Perth, Western Australia, Australia

${ }^{5}$ Centre for Epidemiology and Biostatistics and Melbourne Clinical and Translational Science Platform, University of Melbourne School of Population and Global Health, Melbourne, Victoria, Australia

${ }^{6}$ Department of Anaesthesia, Royal Children's Hospital Melbourne, Melbourne, Victoria, Australia

${ }^{7}$ Murdoch Children's Research Institute, Melbourne, Victoria, Australia

${ }^{8}$ Centre for Health Policy, Melbourne School of Population and Global Health, University of Melbourne, Melbourne, Victoria, Australia

Contributors JD: was involved in study design, protocol drafting and manuscript preparation. BSvU-S: was involved in study design, protocol drafting and manuscript proofing. SB: was involved in study design, protocol drafting and manuscript proofing. DS: was involved in study design, protocol drafting and manuscript proofing. $\mathrm{AD}$ : was involved in study design, protocol drafting and manuscript proofing. MA: was involved in study design, protocol drafting and manuscript proofing. AT-D: was involved in study design, protocol drafting and manuscript proofing. DM: was involved in study design, protocol drafting and manuscript preparation. KL: was involved in study design, protocol drafting and manuscript preparation.

Funding This work has been supported by a project grant from the Australian and New Zealand College of Anaesthetists, and the Victor Hurley Research grant from Melbourne Health. BSvU-S is partly funded by the Perth Children's Hospital Foundation, the Stan Peron Charitable Trust and the Frank Callahan Estate.

Competing interests None declared.

Patient consent for publication Not required.

Ethics approval Ethics approval for thisstudy has been granted by the Melbourne Health Human Research and EthicsCommittee (19 February 2018, HREC/18/MH/2).

Provenance and peer review Not commissioned; externally peer reviewed.

Open access This is an open access article distributed in accordance with the Creative Commons Attribution Non Commercial (CC BY-NC 4.0) license, which permits others to distribute, remix, adapt, build upon this work non-commercially, and license their derivative works on different terms, provided the original work is properly cited, appropriate credit is given, any changes made indicated, and the use is non-commercial. See: http://creativecommons.org/licenses/by-nc/4.0/.

\section{REFERENCES}

1. Weiser TG, Haynes AB, Molina G, et al. Estimate of the global volume of surgery in 2012: an assessment supporting improved health outcomes. Lancet 2015;385(Suppl 2):S11.

2. Apfel CC, Korttila K, Abdalla M, et al. A factorial trial of six interventions for the prevention of postoperative nausea and vomiting. N Engl J Med 2004;350:2441-51.

3. Gan TJ, Diemunsch P, Habib AS, et al. Consensus guidelines for the management of postoperative nausea and vomiting. Anesth Analg 2014;118:85-113.

4. Rodseth RN, Gopalan PD, Cassimjee HM, et al. Reduced incidence of postoperative nausea and vomiting in black South Africans and its utility for a modified risk scoring system. Anesth Analg 2010;110:1591-4.

5. Tennant IA, Augier R, Crawford-Sykes A, et al. Anaesthetic morbidity at the University Hospital of the West Indies. West Indian Med J 2009;58:452-9. 
6. Ugochukwu O, Adaobi A, Ewah R, et al. Postoperative nausea and vomiting in a gynecological and obstetrical population in South Eastern Nigeria. Pan Afr Med J 2010;7:6.

7. Gold BS, Kitz DS, Lecky JH, et al. Unanticipated admission to the hospital following ambulatory surgery. JAMA 1989;262:3008-10.

8. Meeks GR, Waller GA, Meydrech EF, et al. Unscheduled hospital admission following ambulatory gynecologic surgery. Obstet Gynecol 1992;80(3 Pt 1):446-50.

9. Blacoe DA, Cunning E, Bell G. Paediatric day-case surgery: an audit of unplanned hospital admission Royal Hospital for Sick Children, Glasgow. Anaesthesia 2008;63:610-5.

10. Kranke P, Eberhart LH. Possibilities and limitations in the pharmacological management of postoperative nausea and vomiting. Eur J Anaesthesiol 2011;28:758-65.

11. Eberhart $\mathrm{LH}$, Morin $\mathrm{AM}$, Wulf $\mathrm{H}$, et al. Patient preferences for immediate postoperative recovery. Br J Anaesth 2002;89:760-1.

12. Gan TJ, Ing RJ, de L Dear G, et al. How much are patients willing to pay to avoid intraoperative awareness? J Clin Anesth 2003;15:108-12.

13. Kabré YB, Traoré IS, Kaboré FA, et al. Anesthesia for ambulatory pediatric surgery in sub-Saharan Africa: a pilot study in Burkina Faso. Anesth Analg 2017;124:623-6.

14. Frenzel JC, Kee SS, Ensor JE, et al. Ongoing provision of individual clinician performance data improves practice behavior. Anesth Analg 2010;111:515-9.

15. Management Sciences for Health. International medical products price guide. Medford: Management Sciences for Health, 2016.

16. Kim YH, Kim KS, Lee HJ, et al. The efficacy of several neuromuscular monitoring modes at the P6 acupuncture point in preventing postoperative nausea and vomiting. Anesth Analg 2011;112:819-23.

17. Chaiyakunapruk N, Kitikannakorn N, Nathisuwan S, et al. The efficacy of ginger for the prevention of postoperative nausea and vomiting: a meta-analysis. Am J Obstet Gynecol 2006;194:95-9.

18. Frey $\mathrm{UH}$, Scharmann P, Löhlein $\mathrm{C}$, et al. P6 acustimulation effectively decreases postoperative nausea and vomiting in high-risk patients. Br J Anaesth 2009;102:620-5.

19. Short V, Herbert G, Perry R, et al. Chewing gum for postoperative recovery of gastrointestinal function. Cochrane Database Syst Rev 2015;116:Cd006506

20. Darvall JN, Simmons SW, Leslie K. Acceptability of chewing gum for postoperative nausea and vomiting prevention in high risk patients: a pilot study. Anaesth Intensive Care 2011;39:515-6.

21. Chernik DA, Gillings D, Laine $\mathrm{H}$, et al. Validity and reliability of the Observer's Assessment of Alertness/Sedation Scale: study with intravenous midazolam. J Clin Psychopharmacol 1990;10:244-51.
22. Darvall JN, Handscombe M, Leslie K. Chewing gum for the treatment of postoperative nausea and vomiting: a pilot randomized controlled trial. Br J Anaesth 2017;118:83-9.

23. Walker E, Nowacki AS. Understanding equivalence and noninferiority testing. J Gen Intern Med 2011;26:192-6.

24. ANZCA. Professional document: recommendations on monitoring during anaesthesia (PS18). 2017 http://www.anzca.edu.au/ infocentres/downloads/profdocs/index.htm.

25. Hunt R, Dienemann J, Norton HJ, et al. Aromatherapy as treatment for postoperative nausea: a randomized trial. Anesth Analg 2013;117:597-604.

26. Boogaerts JG, Vanacker E, Seidel L, et al. Assessment of postoperative nausea using a visual analogue scale. Acta Anaesthesiol Scand 2000;44:470-4.

27. Harris PA, Taylor R, Thielke R, et al. Research electronic data capture (REDCap)--a metadata-driven methodology and workflow process for providing translational research informatics support. $J$ Biomed Inform 2009;42:377-81.

28. Aspen Pharmacare Australian Pty Ltd. Product information: Zofran injection, tables, syrup, suppositories and Zofran Zydis wafers. Aspen Pharmacare Australian Pty Ltd: St Leonards, 2015.

29. Stark PA, Myles PS, Burke JA. Development and psychometric evaluation of a postoperative quality of recovery score: the QoR-15. Anesthesiology 2013;118:1332-40.

30. OPTUM. SF-12 Health Survey. https://campaign.optum.com/optumoutcomes.html (Accessed 22nd Jan 2017).

31. United States Department of Health and Human Services Food and Drug Administration. Non-inferiority clinical trials to establish effectiveness guidance for industry. 10001 new hampshire ave. Hillandale BIdg, Silver Spring, MD, USA: United States Department of Health and Human Services Food and Drug Administration, 2016.

32. Bodner M, White PF. Antiemetic efficacy of ondansetron after outpatient laparoscopy. Anesth Analg 1991;73:250-4.

33. Du Pen S, Scuderi P, Wetchler B, et al. Ondansetron in the treatment of postoperative nausea and vomiting in ambulatory outpatients: a dose-comparative, stratified, multicentre study. Eur J Anaesthesiol Suppl 1992;6:55-62.

34. Khalil S, Rodarte A, Weldon BC, et al. Intravenous ondansetron in established postoperative emesis in children. S3A-381 Study Group. Anesthesiology 1996;85:270-6.

35. Kazemi-Kjellberg F, Henzi I, Tramèr MR. Treatment of established postoperative nausea and vomiting: a quantitative systematic review. BMC Anesthesiol 2001;1:2. 